\title{
Pulsed Laser Copolymerization of Styrene and Maleic Anhydride
}

\author{
R. A. Sanayei and K. F. O'Driscoll*
}

Institute for Polymer Research, Department of Chemical Engineering, University of Waterloo, Waterloo, Ontario, Canada N2L $3 G 1$

\section{B. Klumperman}

DSM Research, Geleen, The Netherlands

Received April 12, 1994; Revised Manuscript Received June 27, 1994

\begin{abstract}
Pulsed laser polymerization (PLP) has been done for the free radical copolymerization of styrene (monomer 1 ) and maleic anhydride (monomer 2 ) at 25,35 , and $50^{\circ} \mathrm{C}$ in butanone (methyl ethyl ketone (MEK) and acetonitrile (Acn) using AIBN as photoinitiator. The mean values for the propagation rate constant, $\left\langle k_{\mathrm{p}}\right\rangle$, were determined and found to be independent of solvent. Copolymer compositions were measured as a function of comonomer ratio in an isothermal continuous stirred reactor using MEK as solvent over a temperature range from 60 to $140^{\circ} \mathrm{C}$. The combination of these data and the data from PLP experiments were used in model discrimination by multivariate nonlinear regression. The best description of this copolymerization is obtained from the penultimate unit model. No convergence could be reached in a multivariate nonlinear regression of the combined data set to the complex participation model. The mean $k_{\mathrm{p}}$ is well described by the Mayo-Lewis model, where copolymer composition shows some deviation from the model. Numerical inspection reveals that invoking complex participation leads to a good fit of the copolymer composition data but very bad agreement with the mean $k_{\mathrm{p}}$ data. The comonomer pair showed several peculiarities compared to systems described in the literature so far. Extremely high molecular weight copolymer was formed during the PLP experiments, the amount being dependent, among others, on solvent. Furthermore, large amounts of oligomers, i.e., dimers and trimers, are formed under PLP conditions. Two possible explanations for the anomalies of this comonomer pair are discussed. First, diradical initiation could account for the observed phenomena. Laser pulses are thought to excite electron donor-acceptor complexes, resulting in diradicals, which may initiate copolymerization. Second, "dark" polymerization might give rise to formation of high molecular weight copolymer. Due to significant or even total light absorption, part of the monomer solution is unaffected by the laser pulses.
\end{abstract}

\section{Introduction}

The copolymerization of styrene (STY) and maleic anhydride (MAnh) has been shown to deviate from the traditional Mayo-Lewis or terminal model. ${ }^{1}$ However, a conclusive discrimination among more comprehensive models has never been possible. Some work seems to lead toward a preference of the complex participation model, ${ }^{2,3}$ whereas other work points at the penultimate unit model..$^{4,5}$ The main objective of the present work is to use the combination of copolymer composition and PLP data to accomplish conclusive model discrimination between complex participation and the penultimate unit model.

Another goal in this work is to look at the occurrence of diradical initiation. Ten years ago Sadhir et al. ${ }^{6}$ proposed a diradical mechanism for the initiation and propagation in the laser-initiated copolymerization of STY and MAnh. Contrarily, Miner et al. ${ }^{7}$ recently observed that when a $5 \mathrm{MSTY} / \mathrm{MAnh}$ mixture in acetone was pulsed with $365 \mathrm{~nm}$ laser light, $1 \mathrm{~ns} /$ pulse at $60 \mathrm{~Hz}$ for up to an hour, no polymer was formed. They argue that this observation rules out the participation of diradicals in SMA photopolymerization.

\section{Experimental Section}

Pulsed Laser Copolymerization of SMA. STY was washed three times with $10 \% \mathrm{NaOH}$ solution and three times with water to remove inhibitor. It was stored at $-10^{\circ} \mathrm{C}$ after drying over calcium chloride. It was freshly distilled under reduced pressure before use. AIBN was recrystallized twice from absolute methanol. MAnh (Aldrich, 99\% pure), MEK (DBH, AnalaR

\footnotetext{
* To whom correspondence should be addressed.

Abstract published in Advance ACS Abstracts, August 15, 1994.
}

grade), and Acn (Fisher, HPLC grade) were used as received without any further purification.

The experimental setup for the PLP work has been described before by Davis et al. ${ }^{\text {The }}$ The laser light employed here has a wavelength of $355 \mathrm{~nm}$ at a pulse energy of $35 \mathrm{~mJ} / \mathrm{pulse}$ and a pulse width of $15 \mathrm{~ns}$ at half-height.

To test the PLP setup and the SEC calibration, pulsed laser homopolymerization of STY in bulk was carried out at $25^{\circ} \mathrm{C}$ with different pulse rates and different numbers of pulses. AIBN was used as the initiator at a concentration of about $10^{-3} \mathrm{~mol} \mathrm{~L}^{-1}$. The average $k_{\mathrm{p}}$ of four STY PLP experiments was 78.6 (all rate constants are in units of $\mathrm{L} \mathrm{mol} \mathrm{m}^{-1} \mathrm{~s}^{-1}$ ), which is in excellent agreement with the literature values. ${ }^{9}$

Two stock solutions of about $50 \%(\mathrm{w} / \mathrm{w})$ STY and $50 \%(\mathrm{w} / \mathrm{w})$ MAnh in MEK with $10^{-3} \mathrm{~mol} / \mathrm{L}$ AIBN were made and used to prepare mixtures containing varying mole fractions of MAnh. Pulsed laser polymerizations with a pulse frequency of $2 \mathrm{~Hz}$ were performed in sealed quartz glass ampules after degassing the solutions by three successive freeze-pump-thaw cycles. Polymer and residual MAnh were isolated by evaporation of the residual STY and MEK under high vacuum at room temperature.

Initially, samples with more than $5 \mathrm{~mol} \%$ MAnh in the reaction mixture gave polymer which could not be subjected to SEC analysis because the solutions would not pass through the syringe filter. We believe that the samples were of extremely high $\mathrm{MW}$. Even if they could have passed through the filter, the SEC column resolution was such that we could not have done quantitative analyses on them. Subsequent samples were done at higher pulse rates and lower total monomer concentrations and provided copolymer which was amenable to SEC analysis.

SEC Analysis. The multidetector SEC used was equipped with a $60 \mathrm{~cm}$ mix-bed column (Polymer Laboratories) with a 5 $\mu \mathrm{m}$ bead size and with DRI, UV, and on-line viscometer detectors. It was operated at room temperature where HPLC grade THF with $5 \%(v / v)$ acetic acid (glacial grade) was utilized as solvent at a flow rate of $1 \mathrm{~mL} / \mathrm{min}$. Addition of acetic acid to the SEC solvent is required to prevent specific adsorption and/or repulsion of the copolymer to the column material. . $^{10}$ 


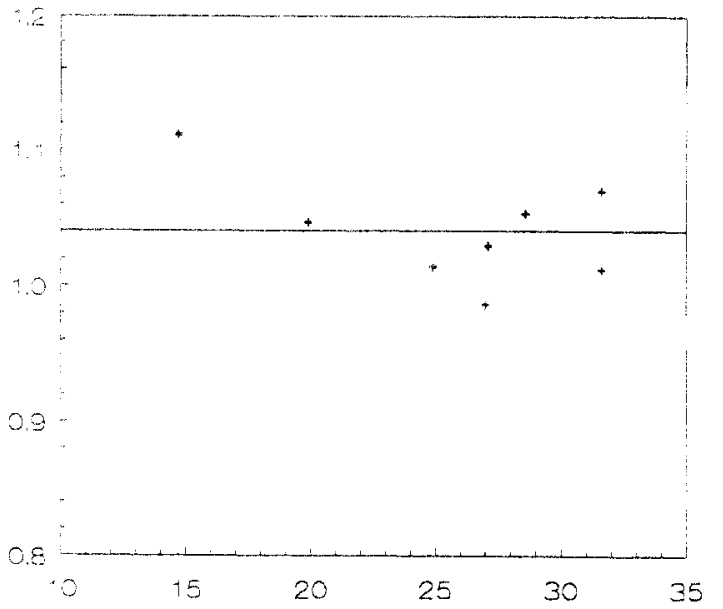

Figure 1. Ratio between $M_{w}$ values determined from the online viscometer and from the DRI detector as a function of MAnh content of the samples. $x$-axis, MAnh content (wt $\%) ; y$-axis, $M_{\mathrm{w}}(\mathrm{visc}) / M_{\mathrm{w}}(\mathrm{DRI})$.

Table 1. Intrinsic Viscosities of PSTY Standards in THF with 5\% (v/v) Acetic Acid

\begin{tabular}{rcrc}
\hline MW & {$[\eta](\mathrm{mL} / \mathrm{g})$} & \multicolumn{1}{c}{ MW } & {$[\eta](\mathrm{mL} / \mathrm{g})$} \\
\hline 1050 & 2.6 & 50000 & 21.9 \\
1770 & 2.6 & 97200 & 35.7 \\
3550 & 4.9 & 200000 & 54.6 \\
7600 & 6.7 & 394000 & 99.4 \\
17000 & 11.3 & 900000 & 158.6 \\
24400 & 13.1 & 1800000 & 293.7
\end{tabular}

Table 2. Estimation of Molecular Weights of SMA Samples from Different Detectors

\begin{tabular}{ccrrrrr}
\hline$F(\mathrm{MAnh})$ & $M_{\mathrm{n}}(\mathrm{DRI})$ & $M_{\mathrm{w}}(\mathrm{DRI})$ & $M_{\mathrm{n}}$ (UV) & $M_{\mathrm{w}}(\mathrm{UV})$ & $M_{\mathrm{n}}$ (visc) & $M_{\mathrm{w}}$ (visc) \\
\hline 0.155 & 41400 & 75500 & 41700 & 78600 & 52600 & 83900 \\
0.209 & 53500 & 94200 & 53300 & 98700 & 63000 & 98600 \\
0.260 & 50000 & 85300 & 46400 & 88400 & 55300 & 86500 \\
0.267 & 65100 & 123500 & 61900 & 130400 & 71500 & 121800 \\
0.283 & 50000 & 91300 & 49000 & 95700 & 56500 & 94000 \\
0.287 & 58400 & 104800 & 57100 & 108700 & 64900 & 110400 \\
0.316 & 96700 & 174000 & 97300 & 185800 & 105100 & 176100 \\
0.329 & 68000 & 127600 & 65600 & 135800 & 79700 & 136500
\end{tabular}

Polystyrene (PSTY) standards were used for an independent primary calibration of each individual detector. The method for independent calibration of detectors ${ }^{11}$ has an important advantage, since it eliminates the estimation of interdetector volume or detector offset. The intrinsic viscosities $([\eta])$ of PSTY standards in THF with $5 \%$ acetic acid were also measured and are reported in Table 1. From these data the $[\eta]-M W$ relationship for PSTY over a discrete molecular weight $(M)$ ranging from 1000 to 1800000 can be represented in the following form: ${ }^{12}$

$$
[\eta]=8.52 \times 10^{-2} M^{1 / 2}+7.35 \times 10^{-5} M
$$

For individual detectors, universal calibration curves in terms of hydrodynamic volume $(M[\eta])$ were established using eq 1 along with primary calibration curves. Then the calibration curve for STY/MAnh copolymers (SMA) was established with a new technique ${ }^{11}$ using only the on-line viscometer and the universal calibration curve. With this technique, the absolute $M_{\mathrm{n}}$ and $M_{\mathrm{w}}$ of (co)polymer samples can be obtained regardless of polymer composition. The results from different detectors are tabulated in Table 2. Figure 1 shows the ratio of absolute $M_{w}$ so determined to the $M_{\mathrm{w}}$ determined from DRI as a function of copolymer composition. From the data in Table 2, the intrinsic viscositymolecular weight relation for SMA copolymers was found to be as follows, independent of copolymer composition over the range studied:

$$
[\eta]=8.724 \times 10^{-2} M^{1 / 2}+10.01 \times 10^{-5} M
$$

As has been discussed a few times in recent publications, ${ }^{13-15}$ the inflection point on the low molecular weight side of the MWD

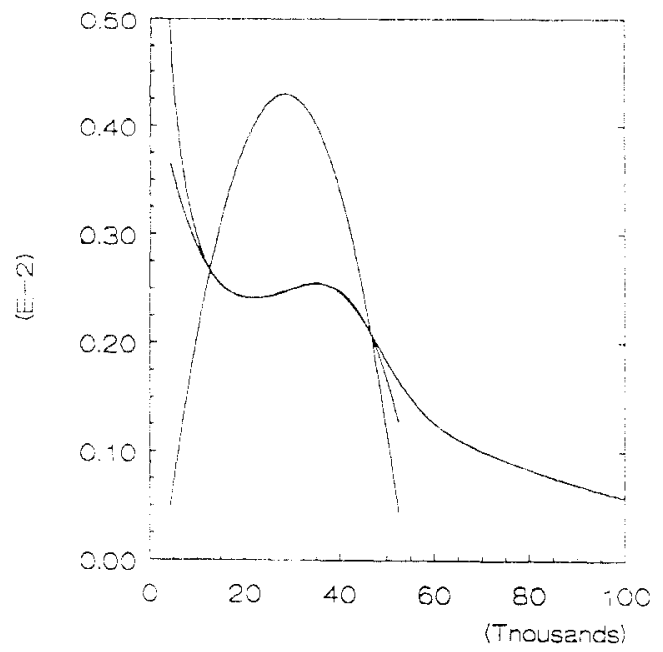

Figure 2. Weight molecular weight distribution of a PLP sample including cubic spline through the relevant part and first derivative thereof. $x$-axis, molecular weight $(M) ; y$-axis, $w(M)$.

is the best measure for the chain length resulting from the repetitive pulsing. To obtain values in accordance with the present "state of the art", the raw SEC data (on a logarithmic scale) were transformed to a linear scale by using the relationship as given in eq 3 for recalculating the ordinate values. ${ }^{16}$

$$
w(M)=\frac{w(\log M)}{M \ln (10)}
$$

where $w(\log M)$ is the weight differential of molecular weight $M$ on a logarithmic plot and $w(M)$ is the corresponding quantity on a linear plot.

The inflection points were determined as maxima in the first derivative of the ordinate with respect to the abscissa in the linear MWD. To obtain maximum accuracy, maxima were determined using a cubic spline through the relevant part of the MWD and calculating the maxima in the analytical derivative. An example of raw data and fitted spline, including the first derivative, all transformed to a linear scale, are shown in Figure 2.

Copolymer Composition. SMA copolymerizations were carried out in an isothermal, continuous stirred tank reactor, using initiators suitable to the temperatures employed, 60,90 , 110 , and $140{ }^{\circ} \mathrm{C}$. This work was similar to that reported previously ${ }_{1}^{17}$ but with the added ability of sampling the reaction mixture directly from the reactor. This allowed the accurate determination of both polymer composition and steady-state conversion, thus enabling the calculation of the comonomer ratio in the reactor at steady state.

\section{Results}

In Figure 3, the results of SMA copolymer composition experiments are shown. The copolymer composition $\left(F_{\text {MAnh }}\right)$ is shown versus the fraction of MAnh $\left(f_{\text {MAnh }}\right)$ relative to the total amount of monomer present at steady state at the temperature studied. The two curves in Figure 3 were calculated using the penultimate unit model with parameters as indicated in the subsequent section (Table 6) at 60 and $140^{\circ} \mathrm{C}$, respectively the minimum and maximum experimental temperature. The calculated curves increase asymptotically to $F_{\mathrm{MAnh}}=0.5$ at $f_{\mathrm{MAnh}}=$ 1.0 , which is caused by the absence of MAnh homopolymerization.

Figure 4 shows the SEC traces $\left(\mathrm{MW}>10^{3}\right)$ for some of the PLP experiments done in pure MEK or MEK/Acn mixtures.

In Tables 3 and 4 , the results of all SMA PLP experiments in MEK solvent are listed. (The sample numbers indicate in sequence the temperature of polymerization, approximate mole percent of MAnh, and 


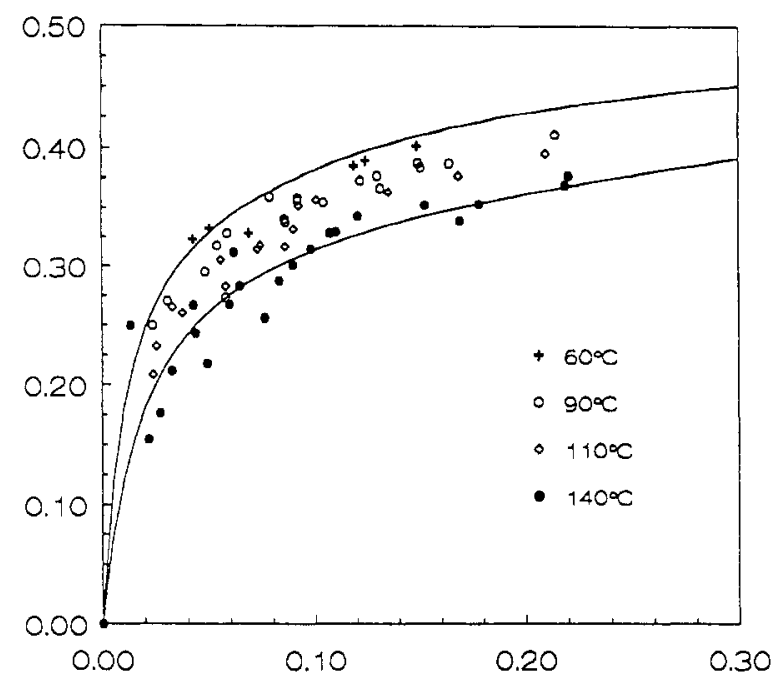

Figure 3. Copolymer composition versus MAnh fraction at steady state from experiments in a continuous stirred tank reactor at $60,90,110$, and $140^{\circ} \mathrm{C}$. $x$-axis, $f_{\text {MAnh }} y$-axis, $F_{\text {MAnh. }}$

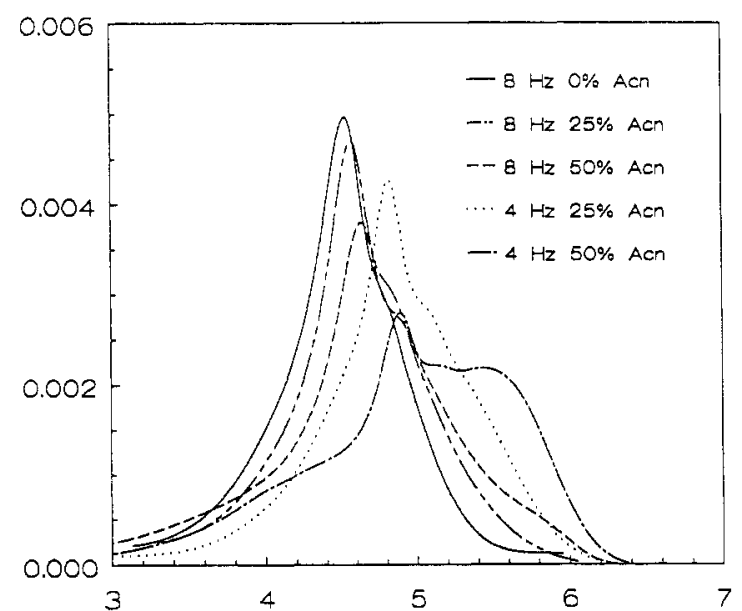

Figure 4. SEC traces (MW $>10^{3}$ ) of PLP samples prepared in MEK and MEK/Acn mixtures at different pulse rates. $x$-axis, $\log (M) ; y$-axis, $w(\log )(M))$

Table 3. SMA Copolymerization by PLP at $25^{\circ} \mathrm{C}$

\begin{tabular}{rccccc}
\hline sample & $\tau(\mathrm{s})$ & $M_{\text {inf }}$ & $f_{\text {MA }}$ & {$[\mathrm{M}]$} & $k_{\mathrm{p}}$ \\
\hline $25-2-1$ & 0.50 & 52685 & 0.02581 & 4.994 & 211.0 \\
$25-2-2$ & 0.25 & 27139 & 0.02581 & 4.994 & 217.4 \\
$25-5-1$ & 0.50 & 65065 & 0.05575 & 3.661 & 355.4 \\
$25-5-2$ & 0.25 & 32448 & 0.05575 & 3.661 & 354.5 \\
$25-5-3$ & 0.50 & 30318 & 0.05575 & 1.836 & 330.3 \\
$25-5-4$ & 0.25 & 15854 & 0.05575 & 1.836 & 345.4 \\
$25-10-1$ & 0.25 & 28219 & 0.11234 & 2.159 & 522.8 \\
$25-10-2$ & 0.50 & 28241 & 0.11234 & 1.083 & 521.5 \\
$25-10-3$ & 0.25 & 14947 & 0.11234 & 1.083 & 552.1 \\
$25-20-1$ & 0.50 & 45029 & 0.19392 & 1.174 & 767.1 \\
$25-20-2$ & 0.25 & 24159 & 0.19392 & 1.174 & 823.1 \\
$25-40-2$ & 0.125 & 50697 & 0.40811 & 2.378 & 1705.5 \\
$25-60-2$ & 0.125 & 50878 & 0.60001 & 1.771 & 2298.3 \\
$25-75-2$ & 0.125 & 49926 & 0.74490 & 1.485 & 2689.6
\end{tabular}

experiment number.) Table 5 shows the data for the PLP experiments done in mixtures of Acn with MEK. In all cases, the mean value of $k_{\mathrm{p}}$ is calculated from the data using the equation

$$
\left\langle k_{\mathrm{p}}\right\rangle=\frac{\nu_{\mathrm{p}}}{\tau([\mathrm{STY}]+[\mathrm{MAnh}])}
$$

where $\nu_{\mathrm{p}}$ is the chain length of copolymer as calculated from the inflection point according to the method outlined above and $\tau$ is the reciprocal of the flashing rate (or the time between two subsequent pulses). For sample 50-
Table 4. SMA Copolymerizations by PLP at 35 and $50^{\circ} \mathrm{C}$

\begin{tabular}{ccrccr}
\hline sample & $\tau(\mathrm{s})$ & \multicolumn{1}{c}{$M_{\text {infl }}$} & $f_{\text {MA }}$ & {$[\mathrm{M}]$} & \multicolumn{1}{c}{$k_{\mathrm{p}}$} \\
\hline $35-10-1$ & 0.250 & 17830 & 0.10818 & 1.084 & 657.9 \\
$35-10-2$ & 0.125 & 7623 & 0.10818 & 1.084 & 562.6 \\
$35-20-1$ & 0.125 & 16003 & 0.19454 & 1.191 & 1074.9 \\
$35-35-1$ & 0.125 & 21691 & 0.33468 & 1.245 & 1393.8 \\
$50-10-1$ & 0.125 & 12904 & 0.10818 & 1.084 & 952.3 \\
$50-20-1$ & 0.125 & 16604 & 0.19454 & 1.191 & 1115.3 \\
$50-35-1$ & 0.125 & $40630^{a}$ & 0.33468 & 1.245 & $2506^{a}$
\end{tabular}

${ }^{a}$ Inflection point and therefore $k_{\mathrm{p}}$ is unreliable as discussed in the text.

Table 5. SMA Copolymerization by PLP in MEK and Acn at $35^{\circ} \mathrm{C}$

\begin{tabular}{cccccc}
\hline MEK/Acn & $\tau$ (s) & $M_{\text {inf }}$ & $f_{\text {MA }}$ & {$[\mathrm{M}]$} & $k_{\mathrm{p}}$ \\
\hline $100 / 0$ & 0.125 & 21691 & 0.33468 & 1.245 & 1393.8 \\
$80 / 20$ & 0.250 & 50753 & 0.33468 & 1.535 & 1322.6 \\
$80 / 20$ & 0.125 & 24886 & 0.33468 & 1.535 & 1297.0 \\
$50 / 50$ & 0.250 & 57325 & 0.33468 & 1.535 & 1493.8 \\
$50 / 50$ & 0.125 & 28309 & 0.33468 & 1.535 & 1475.4
\end{tabular}

35-1 the GPC inflection point could not be discerned by the method described because of a strong background signal. The value given for $M_{\text {infl }}$ is an approximation, estimated from the GPC; it was used to approximate $k_{\mathrm{p}}$, but that value was not used in the regression analysis described below.

\section{Discussion}

Figure 5 shows a plot of the results for the mean $\left\langle k_{\mathrm{p}}\right\rangle$ as a function of the MAnh content of the feed $\left(f_{\text {MAnh }}\right)$ over the entire range of MAnh content studied. Also shown in Figure 5 are the curves as calculated using the penultimate unit effect (PUE) model, using in a modified form the equations set forward by Fukuda. ${ }^{18}$ Using Fukuda's terminology and respecting that MAnh (monomer 2) does not homopolymerize under the conditions of this work, we set $k_{122}$ and $k_{222}$ equal to zero. Therefore, the ratio $r_{2} / k_{22}=1 / k_{121}$, and the mean $\left\langle k_{\mathrm{p}}\right\rangle$ becomes

$$
\begin{gathered}
\left\langle k_{\mathfrak{p}}\right\rangle=\frac{\bar{r}_{1} f_{1}{ }^{2}+2 f_{1} f_{2}}{\left(\bar{r}_{1} f_{1} / \bar{k}_{11}\right)+\left(f_{2} / k_{12}{ }^{1}\right)} \\
\bar{r}_{1}=\frac{r_{21}\left(f_{1} r_{11}+f_{2}\right)}{f_{1} r_{21}+f_{2}} \\
\bar{k}_{11}=\frac{k_{111}\left(r_{11} f_{1}+f_{2}\right)}{r_{11} f_{1}+f_{2} / s_{1}}
\end{gathered}
$$

with $s_{1}$ defined as $k_{211} / k_{111}$.

The parameters needed to model the composition variation of $\left\langle k_{\mathrm{p}}\right\rangle$ according to eq 5 are $k_{111}, k_{121}, k_{211}, r_{11}$ $=k_{111} / k_{112}$, and $r_{21}=k_{211} / k_{212}$. The value of $k_{111}$ was taken from the literature. ${ }^{9}$ The values of $r_{11}, r_{21}, k_{211}$, and $k_{121}$ were determined by multivariate nonlinear least squares (NLLS) regression on the combined data of copolymer composition shown in Figure 3 and the mean $\left\langle k_{\mathrm{p}}\right\rangle$ values as a function of MAnh content shown in Figure 5. In doing so, it was observed that the fit was independent of the value of $k_{121}$ so long as it exceeds $10^{5}$. Table 6 shows the Arrhenius parameters of the various rate constants. The values apply in both MEK and MEK/Acn mixtures. As indicated before, the values from Table 6 were used to plot the solid lines in Figures 3 and 5 . The fit to the experimental data is very satisfactory.

The variance-covariance matrix obtained in the NLLS regression shows that frequency factor and activation energy of each of the parameters are very strongly 


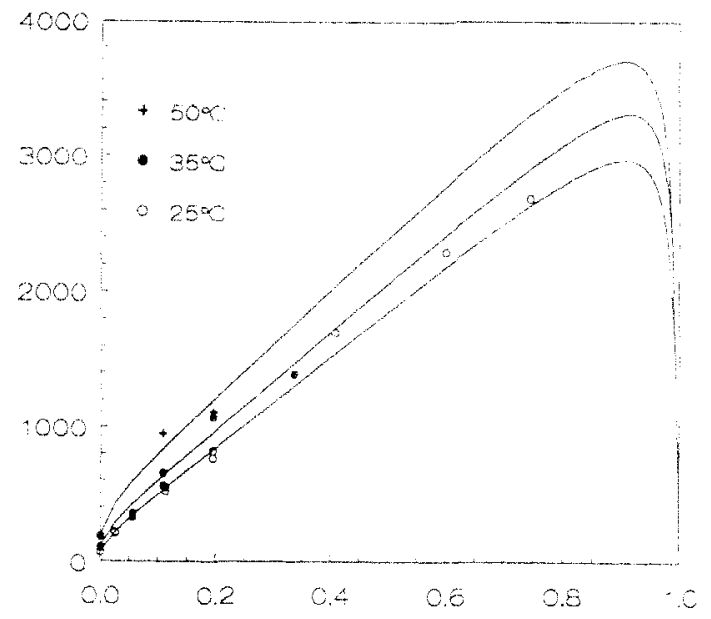

Figure 5. Mean propagation rate constant $\left(k_{\mathrm{p}}\right)$ as a function of monomer feed composition at 25,35 , and $50^{\circ} \mathrm{C}$ with a fit according to the PUE model. $x$-axis, $f_{\text {Manh }} y$-axis, mean $k_{\mathrm{p}}\left(\mathrm{L}, \mathrm{mol}^{-1} \mathrm{~s}^{-1}\right)$.

Table 6. Model Parameters Obtained by Multivariate NLLS Fitting to the PUE Model

\begin{tabular}{ccc}
\hline parameter & $A\left(\mathrm{~L} \mathrm{~mol}^{-1} \mathrm{~s}^{-1}\right)$ & $E\left(\mathrm{~kJ} \mathrm{~mol}^{-1}\right)$ \\
\hline$k_{111^{a}}$ & $1.10 \times 10^{7}$ & 29.5 \\
$k_{211}$ & $3.2 \times 10^{7}$ & 26.1 \\
$r_{11}$ & 0.79 & 9.3 \\
$r_{2 .}$ & 126.5 & 19.6 \\
$k_{121}{ }^{\circ}$ & $>1 \times 10^{\circ}$ &
\end{tabular}

${ }^{a}$ Homopropagation rate constant taken from the literature. ${ }^{9} b$ This parameter had no influence on the fit as long as $k_{121}>10^{5}$.

correlated. Furthermore, $k_{211}$ is strongly correlated with $r_{11}$ and $r_{21}$. Qualitatively this means that there is a large multidimensional confidence interval. Therefore, although the values in Table 6 are the best estimates of the model parameters, no conclusions can be drawn from the absolute numerical values.

In earlier work ${ }^{17}$ it was shown that the ratio $r_{11} / r_{21}$ was sufficient to describe the triad sequence distribution as a function of copolymer composition for the PUE model with the constraint of no homopolymerization of MAnh. In that work we found, mainly based on $110^{\circ} \mathrm{C}$ data, the ratio to be $r_{11} / r_{21}=0.63$. We now find for this ratio a value of approximately 0.2 based on the parameters in Table 6 . Estimation of the error in this value on the basis of the variance-covariance matrix leads to an area as large as -2 $<r_{11} / r_{21}<16$ for the $95 \%$ confidence limits. This provides a good example of the problems arising from the strongly correlated parameters as indicated above. It is probable that better experimental design of experiments may be needed if better estimates of these parameters are to be obtained. Also in the earlier work ${ }^{17}$ it was shown that the "Bootstrap" model was obeyed in the STY/MAnh system. Unfortunately, in this work the solvents studied in the PLP experiments do not provide enough data to comment on the validity of the "Bootstrap" model.

The value of $k_{121}$ cannot be estimated from our data. except to note that it must exceed $10^{5}$. From the Smoluchowski equation we estimate that this rate constant would be $10^{9}$ if it were diffusion controlled. Therefore, the addition of a STY unit to a MAnh radical may be diffusion controlled even at this low conversion.

Besides the PUE model, there have often been attempts to fit SMA data to the monomer complex participation (MCP) model. To accomplish model discrimination between the PUE model and the MCP model, we tried to perform a multivariate regression to the mean $\left\langle k_{\mathrm{p}}\right\rangle$ and copolymer composition data for the MCP model as well. We were unable to obtain a satisfactory fit to both data

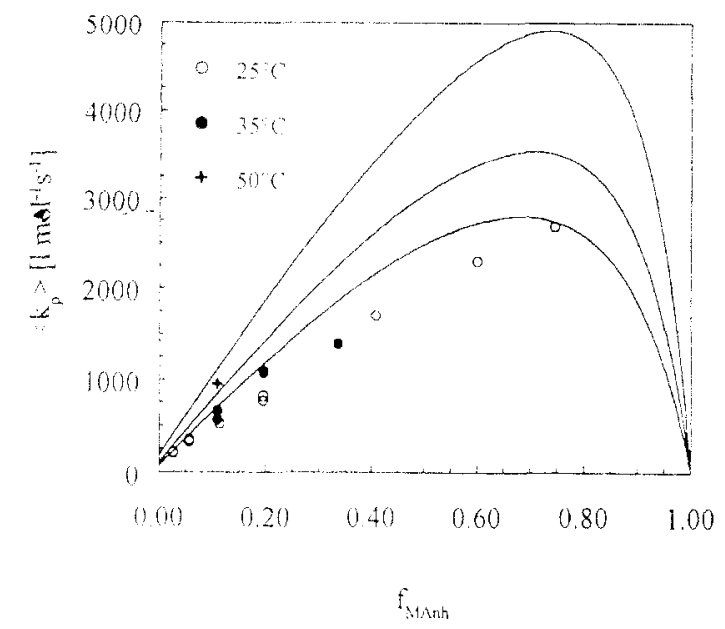

Figure 6. Mean propagation rate constant $\left(k_{\mathrm{p}}\right)$ as a function of monomer feed composition at 25,35 , and $50^{\circ} \mathrm{C}$ with a fit according to the MCP model. $x$-axis, $f_{\text {MAnh; }} y$-axis, mean $k_{\mathrm{p}}\left(\mathrm{L} \mathrm{mol}^{-1} \mathrm{~s}^{-1}\right)$.

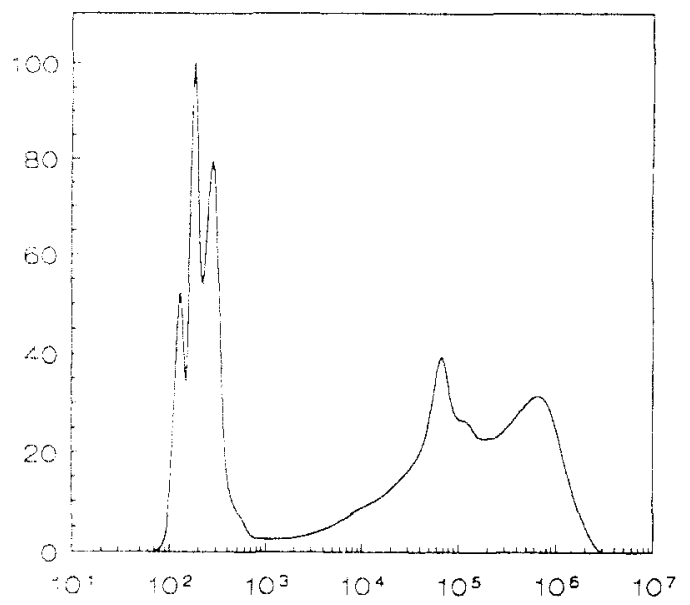

Figure 7. Complete SEC trace of a PLP sample (25-2-1 from Table 3). $x$-axis, $M$; $y$-axis, $w(\log (M))$.

sets using one set of parameters; i.e., no convergence was obtained in the multivariate nonlinear regression to the MCP model. We found that mean $\left\langle k_{\mathrm{p}}\right\rangle$ versus monomer feed composition could be described by the Mayo-Lewis model, which of course is a special case of the MCP model. This parameter set, however, provided a poorer description of the copolymer composition versus monomer feed than did the PUE model. To obtain a satisfactory fit to the Mayo-Lewis model, it was required to invoke some complex addition. In doing so, the maximum in the mean $\left\langle k_{p}\right\rangle$ versus monomer feed shifted toward the equimolar mixture of STY and MAnh, which is obviously not a good description of the experimental points in Figure 5. The shift toward equimolar STY/MAnh composition is easily understood from the background of the MCP, where STY and MAnh are assumed to add as equimolar complexes to the polymer chain. By way of illustration of the poor fit of the MCP model, Figure 6 shows how it describes the experimental mean $k_{\mathrm{p}}$ data.

MWD Anomalies. The SMA PLP experiments exhibited some anomalies as compared to previously described pulsed laser copolymerizations. As can be seen from the SEC trace in Figure 7, high molecular weight polymer and oligomer species are formed during the same experiment. It is obvious to us from work done so far that the SMA/MEK system photoinitiates easily, even in the absence of initiator. This has been observed previously ${ }^{19}$ where it was observed that some solvents facilitated the photopolymerization; e.g., Acn caused a much faster rate of polymerization than did MEK. It was suggested that a charge transfer complex was involved. 
In our PLP work with AIBN initiation, we observed a strange MWD compared to the MWD usually observed in PLP experiments with other monomer systems. We found that changing the frequency of flashing has a significant influence on the amount of a very high MW tail to the observed MWD. The effect of the frequency of flashing appeared to be nonsystematic. Experiments were mainly done in pairs where, within a pair, only the frequency of flashing was varied. In some cases the higher frequency produced the largest amount of high MW polymer, whereas in other examples the lower frequency produced the largest amount of high MW polymer.

We also observed in the absence of AIBN that even higher MW polymer was formed. When we partially substituted Acn for MEK, we observed the peak induced by the pulsed laser to occur at the same chain length (i.e., it gave the same value for the mean $\left.\left(k_{\mathrm{p}}\right\rangle\right)$, but we also observed much more high MW polymer.

From the method of SEC calibration, we were able to estimate the molecular weight of the oligomers formed during the PLP experiments. Their MW corresponds to dimer and trimer species (approximately, MW equals 200 and 300 ).

The observed anomalies can be explained by two different possible causes: (1) diradical initiation and (2) "dark" polymerization.

Postulated Diradical Polymerization. The diradical initiation is thought to occur in the following way. A charge transfer complex formed between the monomers and/or the solvent is excited to the triplet (diradical) state where it initiates by addition of monomer units to each radical. In its early life, the probability is large that its fate is an intramolecular reaction to yield a cyclic (termination by combination) or linear unsaturated (termination by disproportionation) oligomer. After it reaches a certain critical length, the probability of intramolecular reaction rapidly decreases and the two radical chain ends will propagate as though they were separate chains. This continues until one of two events occurs: if chain transfer (probably to monomer) occurs, the diradical becomes a monoradical chain; if two radical chain ends react by combination, then a diradical chain remains a diradical chain if it reacts with another diradical, or it becomes a monoradical chain if it reacts with a monoradical. This means that diradicals in essence only yield a dead chain after two times chain transfer. Thus, the diradical growth and combination with other diradicals is expected to give rise to very high MW polymer besides a large amount of oligomers (i.e., dimers, trimers, and tetramers).

Changing the flashing rates in PLP is observed to have a significant effect on the production of high MW polymer. The principle of PLP is that a large number of growing polymer chains initiated at one pulse are terminated at the next pulse. As outlined above, diradicals are able to survive a large number of combination reactions, as long as a diradical chain combines with another diradical chain. The effect of flashing rate on the amount of high MW polymer produced is difficult to account for but is thought to be related to the relative number of diradicals and monoradicals produced at each laser pulse. If the number of diradicals is large compared to the number of monoradicals, high MW polymer is easily formed. An increase in flashing rate will cause the buildup of a high diradical level, leading to a large amount of high MW polymer. If the number of diradicals is comparable to the number of monoradicals formed at each pulse, the formation of high MW polymer strongly depends on the experimental conditions. In this case, a decrease in flashing rate (a longer dark period) will permit the growing diradicals to grow and combine for a longer period of time, since at the next pulse, the probability of termination by monoradicals is strongly increased, similar to the usual PLP concept.

If the complex that causes photoinitiation of diradicals does not participate in propagation, then any enhancement of the complex is expected to increase the amount of high MW polymer without affecting the observed PLP peak and the value of $\left\langle k_{\mathrm{p}}\right\rangle$ calculated from it.

Substitution of Acn for MEK is known to increase the rate of photopolymerization..$^{19}$ Therefore our observation of the effect of Acn is consistent with the formation of diradicals. It is hypothesized that partial substitution of Acn for MEK leads to a higher concentration of the STY/ MAnh donor-acceptor complex, which is thought to be the precursor of the diradicals.

The formation of very low molecular weight oligomers in large quantities is an additional indication for diradical initiation. As outlined above, the probability of oligomer formation during the early stages of diradical propagation is large.

Postulated Dark Polymerization. Due to significant light absorption of the wavelength generated by the laser, "dark" polymerization could occur. E.g., if light transmittance at $50 \%$ of the total path length is below $1 \%$, the other $50 \%$ effectively "sees" no laser light. Whenever a polymer chain is initiated by this last percent of light, it has practically no chance of being terminated by a primary radical from a subsequent pulse. It can easily grow all the way till it stops by chain transfer. Clearly, this also will give rise to very high $\mathrm{MW}$ polymer.

Again the effect of flashing rate is difficult to explain. Longer dark periods will permit a growing chain in the "dark" zone to diffuse away from the light source, thus reducing its probability of being terminated at a subsequent pulse even further. This will result in more high MW polymer. How a higher flashing rate could lead to an increasing amount of high MW polymer, as observed in some experiments, is not clear yet.

Substitution of Acn for MEK clearly leads to an increasing amount of high MW polymer. The reference experiment where pure MEK is used with for the rest equal conditions shows no high MW tail in the SEC measurement. UV light absorption of Acn at $355 \mathrm{~nm}$ is very limited.

The formation of very low MW oligomers is hard to explain in terms of "dark" polymerization. The STY/ MAnh charge transfer complex is known to undergo DielsAlder reaction on heating. The chemical structure of the oligomers formed during the present experiments was not investigated. Clearly, light absorption leads to one or more reactions of some sort, since polymerization was observed in the absence of initiator, besides the formation of the oligomers. Therefore it is assumed that the formation of oligomers is a direct consequence of the light absorption, although clarification of the mechanism has to await further characterization of the oligomer species.

It is difficult to reconcile the observation by Miner et al. ${ }^{7}$ that repetitive pulsing produced no copolymer. Several experimental conditions were different from the presently used conditions. I.e., we used generally lower monomer concentrations (except for the lowest MAnh fraction), much slower pulsing rate $(2-8 \mathrm{~Hz}$ versus $60 \mathrm{~Hz})$, different wavelength ( 355 versus $365 \mathrm{~nm}$ ), and varying STY/MAnh ratios where Miner et al. seemingly only used equimolar STY/MAnh solutions. It is assumed that under the conditions as used by Miner et al. the formation of oligomers prevails. 


\section{Conclusions}

Model discrimination on the basis of combined experimental data from PLP and copolymer composition studies shows that the SMA copolymerization is best described by the penultimate unit model. The monomer complex participation model provides a fit which is bad compared to the Mayo-Lewis model. Together with earlier work on the triad distribution of SMA, the present results provide conclusive model discrimination in favor of the penultimate unit model.

Anomalies observed in the PLP experiments on SMA copolymerization are, among others, simultaneous formation of high molecular weight copolymer and very low molecular weight oligomers. Diradical polymerization and "dark" polymerization are used to explain the observed phenomena. In doing so, diradical polymerization seems to give a slightly better explanation of the individual observations. Additional work needs to be done to obtain a rigorous proof in favor of one of the hypotheses.

Acknowledgment. Support for K. O'D. by the Natural Sciences and Engineering Research Council of Canada is appreciated. The use of laser facilities of Prof. M. Winnik at the University of Toronto is greatly appreciated. The authors wish to thank Mr. M. Maassen for the synthesis of the copolymers used in the composition studies and Mrs. T. Tiemersma for help in the multivariate nonlinear regression. We thank Prof. A. German and Dr. A. van Herk at the University of Eindhoven for helpful discussions on the formation of high MW polymer during PLP experiments. We also thank DSM Research for financial support and for permission to publish this work.

\section{References and Notes}

(1) Hill,D. J.T.; O'Donnell, J.H.; O'Sullivan, P.W.Macromolecules 1985, 18, 9-17.

(2) Deb, P. C. J. Polym. Sci., Polym. Lett. Ed. 1985, 23, 233-239.

(3) Fujimori, K.; Craven, I. E. J. Polym. Sci., Polym. Chem. Ed. $1986,24,559-565$.

(4) Chen, S. A.; Chang, G. Y. Makromol. Chem. 1986, 187, 15971602.

(5) Ebdon, J. R.; Towns, C. R.; Dodgson, K. J. Mater. Sci., Rev. Macromol. Chem. Phys. 1986, C26, 523-550.

(6) Sadhir, R. K.; Smith, J. D. B.; Castle, P. M. J. Polym. Sci., Polym. Chem. Ed. 1983, 21, 1315-1329.

(7) Miner, G. A.; Meador, W. E.; Chang, C. K. NASA Tech. Memor. $1990,4166$.

(8) Davis, T. P.; O'Driscoll, K. F.; Piton, M. C.; Winnik, M. A. Macromolecules 1989, 22, 2785-2788.

(9) Mahabadi, H. K.; O'Driscoll, K. F. J. Macromol. Sci., Chem. 1977, A11, 967-976.

(10) Tacx, J. C. J. F.; Meijerink, N. L. J. (DSM Research), personal communication.

(11) Suddaby, K. G.; Sanayei, R. A.; Rudin, A.; O'Driscoll, K. F. Makromol. Chem. 1993, 194, 1965-1974.

(12) Sanayei, R. A.; O'Driscoll, K. F.; Rudin, A. ACS Symp. Ser. $1993,521,103$.

(13) Olaj, O. F.; Bitai, I.; Hinkelmann, F. Makromol. Chem. 1987, 188,1689 .

(14) O'Driscoll, K. F.; Kuindersma, M. E. Makromol. Chem., Theory Sim. 1994, 469.

(15) Hutchinson, R. A.; Aronson, M. T.; Richards, J. R. Macromolecules 1993, 26, 6410-6415.

(16) Peebles, L. H. Molecular Weight Distributions in Polymers; Interscience Publishers: New York, 1971.

(17) Klumperman, B.; O'Driscoll, K. F. Polymer 1993, 34, 10321037.

(18) Fukuda, T.; Kubo, K.; Ma, Y.-D. Prog. Polym. Sci. 1992, 17, 875.

(19) Barton, J.; Capek, I.; Arnold, M.; Rätzsch, M. Makromol. Chem. $1980,181,241$. 